\title{
Expression and activity of the urokinase plasminogen activator system in canine primary brain tumors
}

\author{
John H Rossmeisl ${ }^{1-3}$ \\ Kelli Hall-Manning ${ }^{4}$ \\ John L Robertson ${ }^{1,3,5}$ \\ Jamie N King ${ }^{1,2}$ \\ Rafael V Davalos ${ }^{3,5}$ \\ Waldemar Debinski ${ }^{3}$ \\ Subbiah Elankumaran ${ }^{6, \dagger}$ \\ 'Veterinary and Comparative Neuro- \\ Oncology Laboratory, ${ }^{2}$ Department of \\ Small Animal Clinical Sciences, ${ }^{3}$ The \\ Brain Tumor Center of Excellence, \\ Wake Forest Baptist Medical Center \\ Comprehensive Cancer Center, \\ Winston-Salem, NC, ${ }^{4}$ Virginia Tech \\ Animal Laboratory Services, Virginia- \\ Maryland College of Veterinary \\ Medicine, ${ }^{5}$ Department of Biomedical \\ Engineering and Mechanics, Virginia \\ Tech-Wake Forest University School \\ of Biomedical Engineering and \\ Sciences, Virginia Tech, ${ }^{6}$ Department of \\ Biomedical Sciences and Pathobiology, \\ Virginia-Maryland College of \\ Veterinary Medicine, Blacksburg, \\ VA, USA
}

tThe authors regret to advise of the passing of Dr Subbiah Elankumaran prior to publication
This article was published in the following Dove Press journal:

OncoTargets and Therapy

12 April 2017

Number of times this article has been viewed

Background: The expression of the urokinase plasminogen activator receptor (uPAR), a glycosylphosphatidylinositol-anchored protein family member, and the activity of its ligand, urokinase-type plasminogen activator (uPA), have been associated with the invasive and metastatic potentials of a variety of human brain tumors through their regulation of extracellular matrix degradation. Domesticated dogs develop naturally occurring brain tumors that share many clinical, phenotypic, molecular, and genetic features with their human counterparts, which has prompted the use of the dogs with spontaneous brain tumors as models to expedite the translation of novel brain tumor therapeutics to humans. There is currently little known regarding the role of the uPA system in canine brain tumorigenesis. The objective of this study was to characterize the expression of UPAR and the activity of UPA in canine brain tumors as justification for the development of uPAR-targeted brain tumor therapeutics in dogs.

Methods: We investigated the expression of UPAR in 37 primary canine brain tumors using immunohistochemistry, Western blotting, real-time quantitative polymerase chain reaction analyses, and by the assay of the activity of uPA using casein-plasminogen zymography.

Results: Expression of uPAR was observed in multiple tumoral microenvironmental niches, including neoplastic cells, stroma, and the vasculature of canine brain tumors. Relative to normal brain tissues, uPAR protein and mRNA expression were significantly greater in canine meningiomas, gliomas, and choroid plexus tumors. Increased activity of uPA was documented in all tumor types.

Conclusions: UPAR is overexpressed and uPA activity increased in canine meningiomas, gliomas, and choroid plexus tumors. This study illustrates the potential of uPAR/uPA molecularly targeted approaches for canine brain tumor therapeutics and reinforces the translational significance of canines with spontaneous brain tumors as models for human disease.

Keywords: brain tumor, neuro-oncology, dog, animal model, protease, meningioma, glioma

\section{Background}

While the pathophysiology of tumor progression and metastasis is complex, a fundamental step in the process is the ability of neoplastic cells to initiate proteolysis. ${ }^{1}$ Degradation of basement membranes (BM) and the extracellular matrix (ECM) is necessary for cancer cells to breach natural tissue barriers, migrate, invade, and induce microenvironmental remodeling and angiogenesis. ${ }^{1-3}$ A growing body of evidence has indicated that serine proteases and metalloproteinases are key extracellular proteolytic enzymes that are overexpressed in many solid tumors of several species, including cats, dogs, and humans. ${ }^{3-6}$

Activation of plasminogen to form plasmin is a central event in the regulation of extra- and pericellular proteolysis in both physiological and pathological states, as 
plasmin is a nonspecific serine protease capable of degrading most extracellular proteins. ${ }^{3,7}$ There are two types of plasminogen activators (PAs): tissue-type plasminogen activator (tPA) and urokinase-type plasminogen activator (uPA), both of which can catalyze the conversion of the inactive plasminogen zymogen to plasmin, as well as cause the activation of additional protease zymogens and other latent growth factors. ${ }^{6,7}$ The principal role of tPA involves the intravascular generation of plasmin for thrombolysis, whereas UPA is predominantly associated with degradation of the ECM. ${ }^{7}$

The uPA system consists of the serine proteinases uPA and plasmin, two serpin plasminogen activator inhibitors (PAI; PAI-1 and PAI-2), the serpin plasmin inhibitor $\alpha_{2}$-anti-plasmin, and the cell surface $\mathrm{uPA}$ receptor (uPAR), a member of the glycosylphosphatidylinositol (GPI)-anchored protein family. 2,3 Participation of uPAR in tumor invasion and metastasis involves the plasminogen activation dependent breakdown of the ECM and attachment of invasive cells to ECM components, which is mediated by the specific interaction of uPAR with its ligand, uPA. However, uPAR has also been implicated in tumor progression through mechanisms that are independent of plasminogen activation including extracellular signal transduction via interactions with low-density lipoprotein receptor-related protein, integrins, vascular endothelial growth factor, epidermal growth factor receptor, or platelet-derived growth factor receptor., ${ }^{2,8}$

Naturally occurring brain tumors develop in dogs and have been shown to share clinical, phenotypic, and molecular similarities with their human counterparts. ${ }^{9,10}$ This homology has driven interest in use of dogs with spontaneous brain tumors as models for the development and assessment of novel therapeutics. ${ }^{9,10}$ Although treatments targeting components of the uPA system have been investigated in human gliomas and meningiomas, ${ }^{11-13}$ little is currently known about the expression or activity of $\mathrm{UPAR} / \mathrm{uPA}$ in canine brain tumors. We hypothesized that uPAR/uPA would be overexpressed and active in canine brain tumors when compared with normal brain tissues. This would justify further development and evaluation of uPAR/uPA targeted molecular therapies in dogs.

\section{Materials and methods}

\section{Tissue sources}

Brain tumor samples included surgical biopsy or resection specimens, necropsy samples, or archival paraffin-embedded specimens from clinical cases presented to the Veterinary Teaching Hospital of the Virginia-Maryland College of
Veterinary Medicine. Surgical and necropsy specimens were snap frozen in liquid nitrogen or immersed in Allprotect reagent (Qiagen, Valencia, CA, USA), and stored at $-80^{\circ} \mathrm{C}$ within 30 minutes of collection. Normal brain samples (meninges, cerebral cortex, and choroid plexus) were collected from necropsy and archival paraffin-embedded materials from dogs with no clinical, magnetic resonance imaging, or histopathological evidence of brain disease $(n=5)$. Clients provided written consent for their dog's tissues to be banked in a biospecimen repository and used for research purposes (IACUC Approval 13-153-CVM). Clinicopathological data were recorded from each canine subject including the breed, age, sex, tumor location, and analyses performed on each tumor (Table 1). All tumors were classified and graded by veterinary pathologists according to World Health Organization criteria by examination of hematoxylin and eosin (H\&E) and glial fibrillary acidic protein, vimentin, or Olig-2 stained slides prepared from formalin-fixed, paraffin-embedded specimens, as previously reported. ${ }^{9,14,15}$

\section{UPAR immunohistochemistry}

A 5- $\mu \mathrm{m}$ section was cut from each paraffin-embedded block, mounted on positively charged slides, placed in a drying oven at $40^{\circ} \mathrm{C}$ for 30 minutes, and then placed in a drying oven at $65^{\circ} \mathrm{C}$ for 30 minutes. Immunohistochemistry was performed using a procedure modified from a previously described protocol using an automated staining system (BenchMark XT; Ventana Medical Systems, Inc., Tucson, AZ, USA) and an alkaline phosphatase detection method (Enhanced Alkaline Phosphatase Detection Red Kit; Ventana).${ }^{16}$ Epitope retrieval was performed with Cell Conditioning 1 Solution (Ventana) at $96^{\circ} \mathrm{C}$ for 90 minutes, followed by rinsing in reaction buffer (Ventana). Following rinsing, slides were incubated with a murine anti-uPAR antibody (M7294, Clone; Dako, Santa Cruz, CA, USA; 1:25 dilution using antibody dilution buffer; Ventana) at $37^{\circ} \mathrm{C}$ for 2 hours, and then rinsed three times for 2 minutes each with reaction buffer. Slides were subjected to automated amplification (Amplification Kit; Ventana) for 16 minutes and endogenous biotin blocking (Blocking Kit; Ventana) for 8 minutes prior to the additional of the secondary biotinylated goat antimouse/antirabbit antibody for 30 minutes, and rinsed with reaction buffer. Slides were incubated with a streptavidin-alkaline phosphatase conjugate in Tris buffer with $\mathrm{MgCl}_{2}$ and $\mathrm{ZnCl}$ (Ventana) for 30 minutes. Slides were then developed with a naphthol substrate and fast-red chromogen (Fast Red; Ventana) for 15 minutes, counterstained with Richard-Allan Hematoxylin, and dried at room temperature. Negative controls were performed by 
Table I Clinicopathological characteristics of dogs with primary brain tumors and normal controls

\begin{tabular}{|c|c|c|c|c|c|c|c|c|}
\hline \multirow{2}{*}{$\begin{array}{l}\text { Sample } \\
\text { number }\end{array}$} & \multirow{2}{*}{$\begin{array}{l}\text { Sample } \\
\text { ID }\end{array}$} & \multirow[t]{2}{*}{ Breed, sex, age (years) } & \multirow[t]{2}{*}{ Tumor location } & \multirow[t]{2}{*}{ Diagnosis } & \multicolumn{4}{|c|}{ Analyses performed } \\
\hline & & & & & IHC & WB & ZYM & PCR \\
\hline I & Cll.I & Brittany Spaniel, MN, 2 & Lateral ventricle & Choroid plexus papilloma (Grade II) & Yes & No & Yes & Yes \\
\hline 2 & CII.2 & Golden Retriever, FS, 8 & Cerebellopontomedullary angle & & Yes & Yes & Yes & Yes \\
\hline 3 & CIII.I & Mixed breed, FS, 2 & Cerebellopontomedullary angle & Choroid plexus carcinoma (Grade III) & Yes & No & No & Yes \\
\hline 4 & Menl.I & Min. Schnauzer, FS, 12 & Cerebral convexity & Grade I meningioma & Yes & Yes & No & No \\
\hline 5 & Menl.2 & Mixed breed, FS, 12 & Parasagittal & & Yes & Yes & Yes & Yes \\
\hline 6 & Menl.3 & Mixed breed, FS, I5 & Basilar & & Yes & Yes & Yes & Yes \\
\hline 7 & Menl.4 & Rat Terrier, MN, II & Olfactory & & Yes & No & Yes & No \\
\hline 8 & Menl.5 & Llewellin Setter, MN, II & Falcine & & Yes & Yes & No & No \\
\hline 9 & Menl.6 & Boxer, MN, II & Cerebellar convexity & & Yes & Yes & Yes & Yes \\
\hline 10 & Menl.7 & Keeshond, MN, 8 & Parasellar & & Yes & Yes & Yes & Yes \\
\hline 11 & Menl.8 & Siberian Husky, MN, 7 & Cerebral convexity & & Yes & No & Yes & No \\
\hline 12 & Menl.9 & Am. Staff. Terrier, FS, II & Parasagittal & & Yes & Yes & Yes & Yes \\
\hline 13 & Menl.I0 & Chow, MN, 5 & Cerebellopontomedullary angle & & Yes & Yes & Yes & Yes \\
\hline 14 & Menl.II & Mixed breed, MN, I3 & Unknown & & Yes & Yes & Yes & Yes \\
\hline 15 & Menl.I2 & German Shepherd, FS, 8 & Cerebral convexity & & Yes & No & Yes & Yes \\
\hline 16 & Menl.I3 & Australian Shepherd, F, 7 & Parasagittal & & Yes & No & No & Yes \\
\hline 17 & Menl.I4 & Labrador Retriever, MN, 9 & Cerebellar convexity & & Yes & No & No & No \\
\hline 18 & Menll.I & Mixed breed, MN, 6 & Cerebral convexity & Grade II meningioma & Yes & No & No & Yes \\
\hline 19 & Menll.2 & Golden Retriever, MN, 8 & Parasellar & & Yes & Yes & Yes & Yes \\
\hline 20 & Menll.3 & Whippet, MN, 10 & Falcine & & Yes & Yes & Yes & Yes \\
\hline 21 & Menll.4 & German Shepherd, MN, 12 & Basilar & & Yes & Yes & Yes & Yes \\
\hline 22 & Menll.5 & Mixed breed, FS, II & Falcine & & Yes & No & Yes & Yes \\
\hline 23 & Menll.6 & Boxer, FS, 10 & Olfactory & & Yes & No & No & Yes \\
\hline 24 & Menll.7 & Golden Retriever, FS, 12 & Cerebral convexity & & Yes & Yes & Yes & Yes \\
\hline 25 & Astroll.I & Bullmastiff, FS, 6 & Frontal lobe & Grade II astrocytoma & Yes & Yes & No & Yes \\
\hline 26 & Astroll.2 & Boxer, MN, 7 & Frontoparietal lobe & & Yes & Yes & Yes & Yes \\
\hline 27 & Astrolll.I & Am. Staff. Terrier, MN, 6 & Parietal lobe & Grade III astrocytoma & Yes & Yes & Yes & Yes \\
\hline 28 & GBM.I & Beagle, FS, 6 & Bilateral cerebral (butterfly) & Grade IV astrocytoma & Yes & Yes & Yes & Yes \\
\hline 29 & GBM.2 & Boxer, FS, II & Parieto-temporal lobes & & Yes & Yes & No & No \\
\hline 30 & GBM.3 & Boston Terrier, MN, 2 & Temporal lobe & & Yes & No & Yes & Yes \\
\hline 31 & GBM.4 & Boxer, MN, 8 & Thalamus & & Yes & Yes & Yes & Yes \\
\hline 32 & Oligoll.I & Boxer, MN, 9 & Piriform lobe & Grade II oligodendroglioma & Yes & Yes & Yes & Yes \\
\hline 33 & Oligoll.2 & Boxer, MN, 5 & Fronto-olfactory lobes & & Yes & Yes & No & No \\
\hline 34 & Oligoll.3 & Mixed breed, FS, 8 & Parietal lobe & & Yes & No & Yes & Yes \\
\hline 35 & OligollI.I & Boston Terrier, MN & Piriform lobe & Grade III oligodendroglioma & Yes & No & Yes & Yes \\
\hline 36 & Oligolll.2 & English Bulldog, FS, 7 & Temporal lobe & & Yes & Yes & Yes & Yes \\
\hline 37 & Oligolll.3 & Am. Bulldog, FS, 6 & Frontal lobe & & Yes & Yes & Yes & Yes \\
\hline 38 & Contl & Beagle, FS, 5 & NA & Normal brain & Yes & Yes & Yes & Yes \\
\hline 39 & Cont2 & Beagle, MN, 6 & NA & Normal brain & Yes & Yes & Yes & Yes \\
\hline 40 & Cont3 & Hound Mix, MN, 7 & NA & Normal brain & Yes & Yes & Yes & Yes \\
\hline 41 & Cont4 & Am. Staff. Terrier, MN, 5 & NA & Normal brain & Yes & Yes & Yes & Yes \\
\hline 42 & Cont5 & Mixed breed, FS, 4 & NA & Normal brain & Yes & Yes & Yes & Yes \\
\hline
\end{tabular}

Abbreviations: Am, American; F, female; FS, female spayed; IHC, immunohistochemistry; Min, miniature; MN, male, neutered; NA, not applicable; PCR, polymerase chain reaction; Staff, Staffordshire; WB, Western blotting; ZYM, casein-plasminogen zymography.

substituting the primary antibody with antibody dilution buffer (Ventana). Canine tonsils and intestines were used as the positive control tissue. ${ }^{16}$

\section{uPAR Western blotting (WB)}

Canine tumor lysates were prepared in an extraction buffer (150 mM NaCl, $20 \mathrm{mM}$ Tris/HCl [pH 7.4], 0.1\% sodiumdodecyl sulfate [SDS], $1 \%$ sodium deoxycholate, $1 \%$ Triton $\mathrm{X}-114$ with proteinase inhibitory mixture $[5 \mu \mathrm{g} / \mathrm{mL}$ aprotinin,
$1 \mu \mathrm{g} / \mathrm{mL}$ leupeptin, $1 \mu \mathrm{g} / \mathrm{mL}$ trypsin inhibitor, and $1 \mathrm{mM}$ phenylmethylsulfonyl fluoride]) and incubated for 5 minutes at $37^{\circ} \mathrm{C}$. Extracts were then centrifuged at $2500 \times \mathrm{g}$ for 5 minutes to separate a detergent phase that contains mainly hydrophobic membrane proteins including GPI-anchored uPAR. Human HL-60 leukemia cell extracts prepared in SDS-polyacrylamide gel electrophoresis (PAGE) buffer (Enzo, Farmingdale, NY, USA) were used as a WB-positive control. The total protein concentration was obtained using 
a bicinchoninic assay kit (BCA-1; Sigma-Aldrich, St Louis, MO, USA), and $20 \mu \mathrm{g}$ of protein from these samples was separated under nonreducing conditions by $15 \%$ SDS-PAGE and transferred to nitrocellulose membranes (Bio-Rad, Hercules, CA, USA). After blocking with phosphate-buffered saline-Tween and 5\% nonmilk fat at room temperature for 2 hours, the membrane was blotted with a monoclonal murine anti-uPAR antibody (SAB4200412, 1:500 dilution; Sigma, St Louis, MO, USA) for 1 hour at room temperature. According to the manufacturer, this antibody is cross-reactive with human and canine uPAR. The membrane was rinsed and incubated with goat antimouse horseradish peroxidase secondary antibody (1:1,000 dilution; BD Bioscience, San Jose, CA, USA) for 1 hour at room temperature and developed using an enhanced chemiluminescence detection kit (GE Healthcare Life Sciences, Piscataway, NJ, USA) to allow for relative quantification of uPAR. A rabbit anti- $\beta$-actin polyclonal antibody (Biovision, Mountain View, CA, USA) was used as a protein loading control. Quantitative analyses were performed using a high-resolution digital imaging (Amersham 600; GE Healthcare) and dedicated chemiluminescent software system (Melanie, 2D Gel Package; GE Healthcare).

\section{UPAR real-time quantitative polymerase chain reaction ( $R T-q P C R$ )}

mRNA from canine tumors and brain tissues was isolated using an RNeasy kit (Qiagen) according to the manufacturer's instructions, and all $\mathrm{mRNA}$ samples were stored at $-80^{\circ} \mathrm{C}$ before analyses. An iTaq Universal one-step kit (Bio-Rad) was used to perform the RT-qPCR, with $\beta$-actin serving as an internal control. The primers for uPAR (NCBI-ID XM_014119899.1) were 5'-GCCTTACCGAGGTTGTGTGT-3' (forward) and 5'-CATCCAGGCACTGTTCTTCA-3' (reverse). The $\beta$-actinspecific primers were $5^{\prime}$-CTGGAACGGTGAAGGTGACA-3' (forward) and 5'-GGGAGAGGACTGGGCCATT-3' (reverse). The uPAR primers used in this study were created using the Primer3Plus platform. ${ }^{17}$ The specificity of the uPAR primers and their corresponding amplicons were evaluated in silico using the BLAST online tool. ${ }^{18} \mathrm{uPAR}$ mRNA expression was calculated as the fold-change relative to the $\beta$-actin control using comparative $\mathrm{C}_{\mathrm{T}}$ methodology. ${ }^{9}$

\section{uPA activity: casein-plasminogen zymography}

The activity of uPA in tissues was analyzed by caseinplasminogen zymography using a previously described method. ${ }^{19}$ Tumor homogenates $(10 \mu \mathrm{g}$ protein/lane) were separated by electrophoresis in $10 \%$ polyacrylamide gel copolymerized with $1 \mathrm{mg} / \mathrm{mL}$ of casein (Sigma) and $13 \mu \mathrm{g} / \mathrm{mL}$ human plasminogen (Sigma) under nonreducing conditions. Following electrophoretic separation, the gels were washed twice in $2.5 \%$ Triton-X 100 for 30 minutes, incubated with reaction buffer $(50 \mathrm{mM}$ Tris- $\mathrm{HCl}, 5 \mathrm{mM}$ $\mathrm{CaCl}_{2}$, and $0.02 \% \mathrm{NaN}_{3}, \mathrm{pH} 8.0$ ) at $37^{\circ} \mathrm{C}$ for 18 hours, and then stained with Coomassie blue (Sigma). Purified, highmolecular weight (50 kDa) canine uPA (DUPA; Molecular Innovations, Novi, MI, USA) and human tPA (ab92637; Abcam, Cambridge, UK) were used as positive controls, and negative controls consisted of samples loaded on gels devoid of plasminogen. Specific inhibition of uPA activity was performed by running parallel gels, which contained $2 \mathrm{mM}$ amiloride (Sigma). Amiloride is a selective inhibitor of uPA but does not affect other PAs. ${ }^{20}$

\section{Statistical analysis of WB and RT-qPCR}

To test the null hypothesis that tumor type does not have an effect on uPAR expression, data were modeled by use of a Kruskal-Wallis one-way analysis of variance. To test the null hypothesis that tumor grade does not have an effect on UPAR expression, Jonckheere trend tests were done to compare tumor grade variability within tumor types. When significant differences were identified, Mann-Whitney tests were used for pair-wise comparisons. A Bonferroni's multiple comparison adjustment was used to confirm significant results. For all analyses, alpha was set at 0.05 .

\section{Results \\ Canine tissue samples}

A total of 37 primary canine brain tumor samples were included in analyses of uPAR expression and uPA activity (Table 1). Of 37 samples, 21 were meningiomas, (Grade I, $\mathrm{n}=14$; Grade II, $\mathrm{n}=7$ ), 7 were astrocytomas (Grade II, $\mathrm{n}=2$; Grade III, n=1; and Grade IV [glioblastoma; GBM], n=4), 6 were oligodendrogliomas (Grade II, $\mathrm{n}=3$; Grade III, $\mathrm{n}=3$ ), and 3 were choroid plexus tumors (Grade II, $\mathrm{n}=2$; Grade III, $\mathrm{n}=1$ ).

\section{uPAR immunohistochemistry}

In normal brain tissue controls (Figure 1A-F), uPAR immunoreactivity was observed in rare microglia (Figure 1D) and vascular endothelium contained within the cerebral cortex, as well as consistently in the choroid plexus vascular endothelium (Figure 1F). No uPAR immunoreactivity was observed in normal meninges (Figure 1B).

The neoplastic epithelium in each of the choroid plexus tumors (Figure $1 \mathrm{G}$ and $\mathrm{H}$ ) examined displayed diffuse intense 


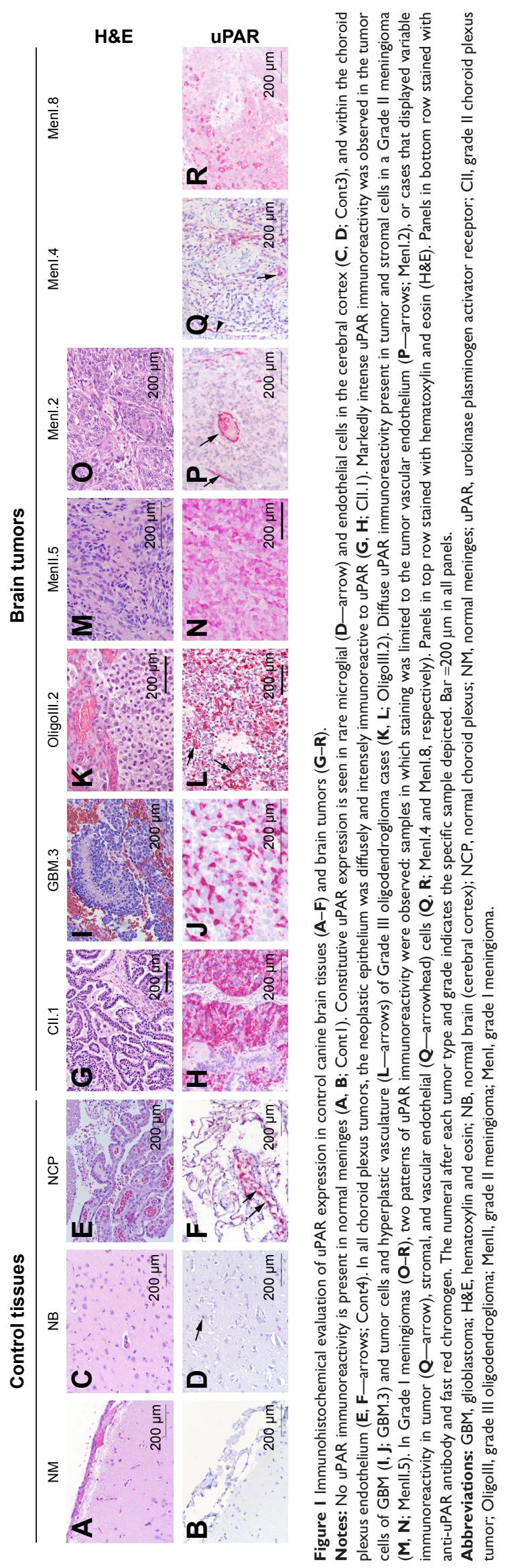

cytoplasmic uPAR immunoreactivity. Immunoreactivity of supporting fibrovascular stromal cells was observed only in the choroid plexus carcinoma.

All astrocytomas (7/7) and oligodendrogliomas (6/6) were immunoreactive to uPAR (Figure 1I-L). Tumor cells in oligodendrogliomas and astrocytomas demonstrated cytoplasmic patterns of uPAR immunoreactivity. In all high-grade glioma samples, $>30 \%$ of neoplastic cells were immunoreactive to uPAR (Figure $1 \mathrm{~J}$ and $\mathrm{L}$ ). The tumor vasculature within both astrocytomas and oligodendrogliomas demonstrated regionally variable immunoreactivity to uPAR but was universally marked in hyperplastic tumor vasculature (Figure 1L). In the two necropsy-derived GBM specimens, the most intense staining of the neoplastic cells and tumor vasculature was observed adjacent to areas of tumor invasion of the neuropil (data not shown).

Among meningiomas (Figure 1M-R), 19 of 21 demonstrated immunoreactivity to uPAR, including 12 of 14 Grade I tumors and 7 of 7 Grade II tumors. In 6 of 14 Grade I meningiomas, uPAR immunoreactivity was predominantly localized to the endothelial cytoplasm within the tumor vasculature (Figure 1P), with $<5 \%$ of neoplastic cells demonstrating UPAR immunoreactivity in these cases. In 6 of 14 Grade I and 7 of 7 Grade II meningiomas, mildly to markedly intense cytoplasmic immunoreactivity was observed in tumor, stromal, and vascular endothelial cells. In Grade I meningiomas, uPAR immunoreactivity in these tumor cellular populations was mild and multifocal in 4 of 6 cases (Figure 1Q and R), whereas in Grade II meningiomas uPAR immunoreactivity was intense and diffuse in 4 of 7 cases (Figure 1N).

\section{UPAR WB}

WB documented expression of UPAR protein in all control and tumor tissues examined. In the positive HL-60 control as well as in some of the canine normal brain and tumor samples, two distinct bands were detected (Figure 2A), one at a MW of $52 \mathrm{kDa}$ and another at $46 \mathrm{kDa}$, corresponding to UPAR isoforms 1 and 3, respectively. Compared with normal brain tissues (Figure $2 \mathrm{~B}$ ), the expression of uPAR was significantly greater in all types of brain tumors, and UPAR expression in GBM was higher compared with all other tumor types and grades.

\section{uPAR RT-qPCR}

Patterns of uPAR gene expression were similar to what was observed in WB analyses, with uPAR expression in all types and grades of brain tumors being significantly 
A

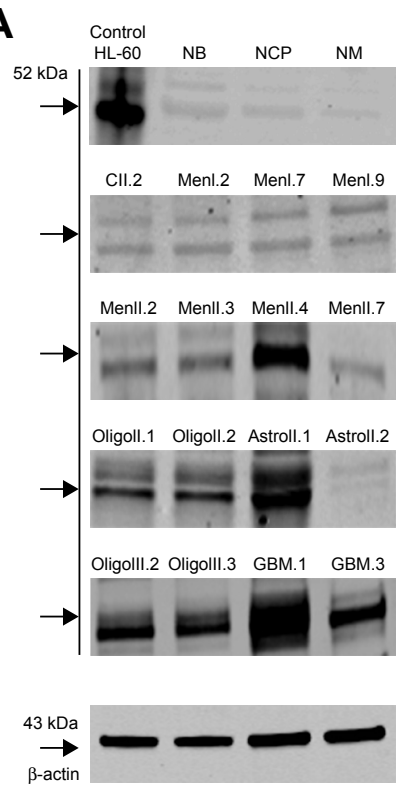

B

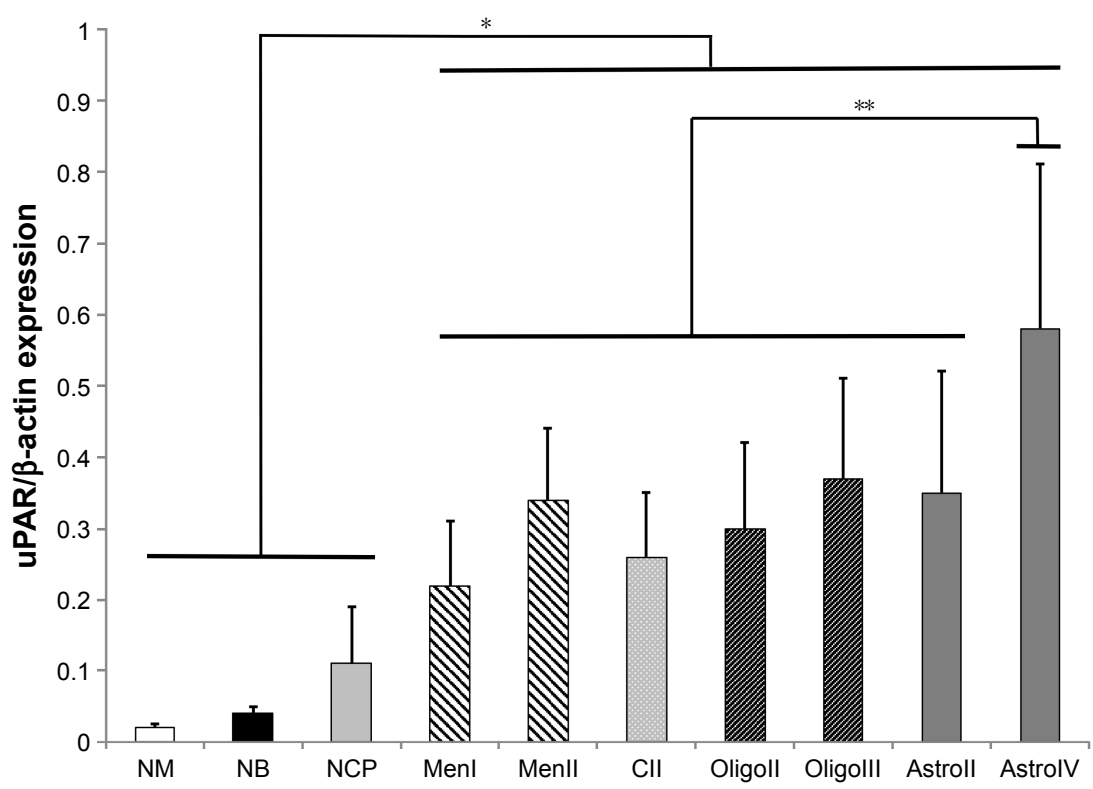

Figure 2 (A) Representative Western blots of uPAR protein in canine normal brain tissues and brain tumors. The numeral after each tumor type and grade indicates the specific sample depicted. (B) uPAR protein expression compared with $\beta$-actin concentrations in normal canine brain tissues and tumors.

Notes: uPAR expression is significantly greater in tumors compared with controls $\left({ }^{*} P<0.02\right)$ and significantly higher in GBM compared with all other tumor types and grades $(* * P<0.001)$.

Abbreviations: GBM, glioblastoma; NM, normal meninges; NB, normal brain (cerebral cortex); NCP, normal choroid plexus; uPAR, urokinase plasminogen activator receptor; Menl, grade I meningioma; Menll, grade II meningioma; CII, grade II choroid plexus tumor; Oligoll, grade II oligodendroglioma; Oligolll, grade III oligodendroglioma; Astroll, grade II astrocytoma; AstrolV, grade IV astrocytoma.

increased compared with control brain tissues $(P<0.002)$. The highest uPAR gene expression was seen in high-grade gliomas (Figure 3). Expression of UPAR in astrocytomas and oligodendrogliomas was higher compared with meningiomas $(P<0.02)$ and choroid plexus tumors $(P<0.04)$. No significant differences in uPAR expression were observed between meningiomas and choroid plexus tumors ( $P=0.43$ ), or between astrocytomas and oligodendrogliomas $(P=0.22)$. Differences in uPAR expression among tumor grades were observed, with expression in GBM being significantly greater than in Grade II and III astrocytomas (combined, $P=0.004$ ), and expression in Grade III oligodendrogliomas was also greater than in Grade II oligodendrogliomas $(P=0.009)$. No significant differences in uPAR gene expression were observed between grades of meningiomas $(P=0.67)$.

\section{uPA activity: casein-plasminogen zymography}

Casein-plasminogen zymography demonstrated lysis bands at molecular weights of $45-55 \mathrm{kDa}$, corresponding to uPA, and $65-70 \mathrm{kDa}$, identified as tPA, in all tissue samples (Figure 4A and B). In the presence of amiloride, inhibition of uPA lysis bands occurred at molecular weights of $\sim 45-60 \mathrm{kDa}$, indicating specific uPA proteolytic activity in all tissue samples (Figure 4C). Qualitatively, uPA activity was detected in all tumor samples, with comparably little activity detected in normal brain tissues.

\section{Discussion}

Meningiomas, gliomas, and choroid plexus tumors account for $\sim 50 \%, 35 \%$, and $10 \%$ of all canine primary brain tumors, respectively. ${ }^{21-23}$ Similarities in the clinical behavior, morphology, molecular signatures, and genetic aberrations have been observed between human and canine brain tumors, which has resulted in the emergence of the brain tumor bearing $\operatorname{dog}$ as a model for translational research., ${ }^{9,10,24}$ While these tumors represent a phenotypically, clinically, and genomically heterogeneous group of cancers, a shared pathophysiological denominator that contributes to the challenges associated with their treatment is their ability to invade the brain parenchyma. ${ }^{8}$ In this study, using several complementary methods, we have demonstrated that uPAR protein and mRNA are overexpressed and uPA activity is present in the most commonly encountered canine primary brain tumors.

Dysregulation of the uPAR interactome has been implicated as an important component of the pathogenesis of numerous human central nervous system disorders including Alzheimer's disease, autism, brain tumors, and epilepsy. ${ }^{24}$ 


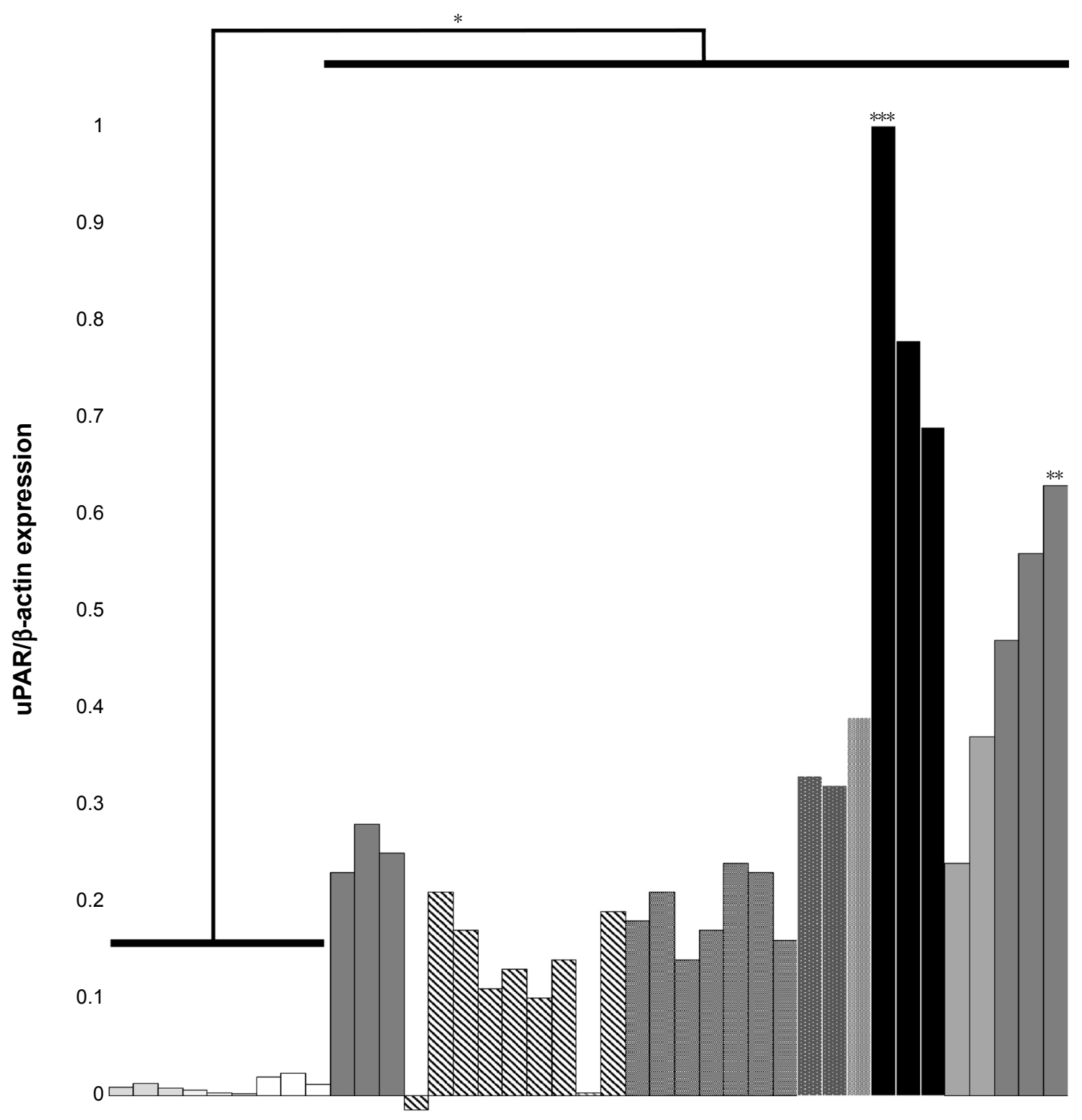

$-0.1$

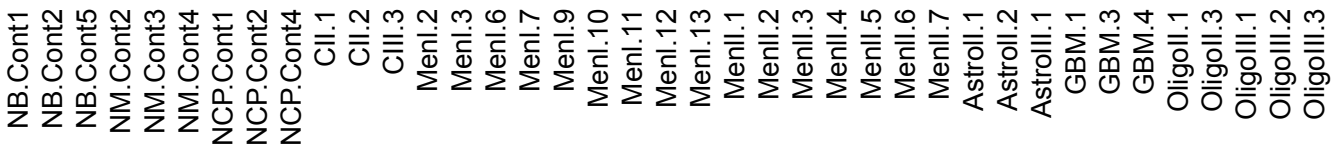

Figure 3 Expression of urokinase plasminogen activator receptor (UPAR) mRNA in canine primary brain tumors as determined by real-time quantitative polymerase chain reaction.

Notes: Expression of uPAR is increased in brain tumors $\left({ }^{*} P<0.02\right)$ compared with normal brain tissues. Among tumors, uPAR expression was significantly higher in glioblastoma $(* * * P<0.003)$ and Grade III oligodendrogliomas $(* * P<0.0 I)$. The numeral after each designation indicates the specific sample depicted.

Abbreviations: NB.Cont, normal brain control; MN.Cont, normal meninges control; NCP.Cont, normal choroid plexus control; CIl, grade II choroid plexus tumor; CIII, grade III choroid plexus tumor; Menl, grade I meningioma; Menll, grade II meningioma; Astroll, grade II astrocytoma; AstrollI, grade III astrocytoma; GBM, glioblastoma; Oligoll, grade II oligodendroglioma; Oligolll, grade III oligodendroglioma.

In human primary brain tumors, UPAR participates in numerous plasminogen activation dependent cell surface oncogenic processes including cellular invasion, migration, and distant metastatic seeding that are mediated via degradation of the BM and ECM. ${ }^{1,13,25}$ Through its interactions with integrins, uPAR also regulates cell-signaling mechanisms involved in tumor cell proliferation, chemotaxis, angiogenesis, and protection from apoptosis. ${ }^{2,25}$ For systemic malignancies, it has also recently been hypothesized that UPAR upregulation in circulating tumor cells may be the 


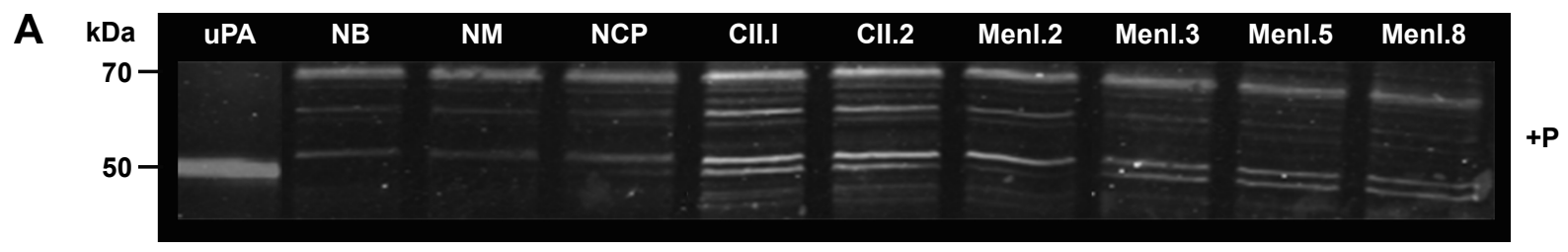

B

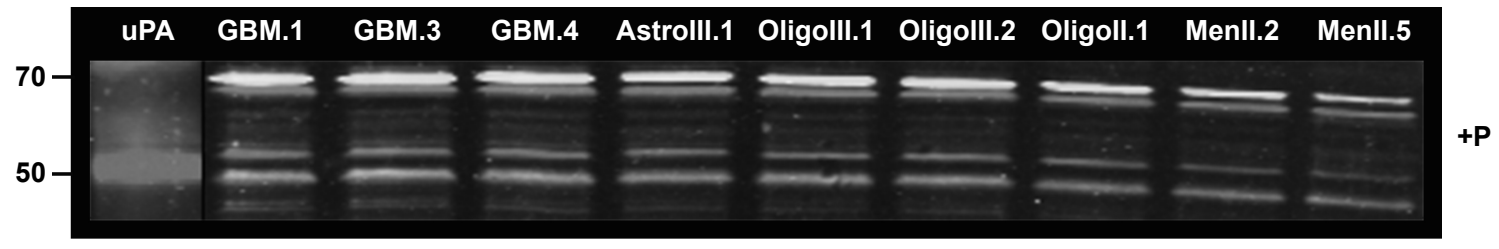

C

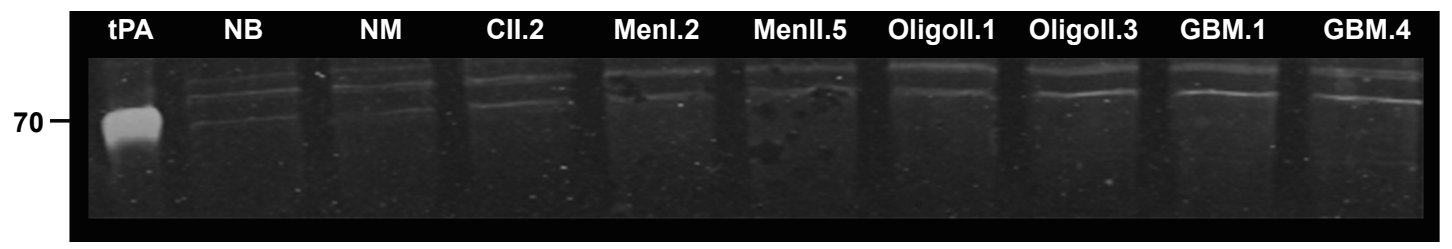

Figure 4 uPA activity in normal canine brain tissues and brain tumors as determined with casein-plasminogen zymography.

Notes: (A, B) Zones of lysis spanning the $45-55 \mathrm{kDa}$ region correspond with uPA activity, which is present in all tumor types and grades. (C) Lysis inhibition of uPA occurs in the presence of the amiloride-treated gels $(+\mathrm{P} /+\mathrm{A})$, with $\mathrm{tPA}$ activity visible at $\sim 70 \mathrm{kDa}$. The numeral after each tumor type and grade indicates the specific sample depicted.

Abbreviations: NB, normal brain; NCP, normal choroid plexus; NM, normal meninges; +P, plasminogen-treated gel; Menl, grade I meningioma; Menll, grade II meningioma; CII, grade II choroid plexus tumor; Oligoll, grade II oligodendroglioma; OligollI, grade III oligodendroglioma; Astroll, grade II astrocytoma; AstrollI, grade III astrocytoma; GBM, glioblastoma; +P/+A, plasminogen and amiloride-treated gel; tPA, purified human tissue plasminogen activator control; uPA, urokinase-type plasminogen activator control.

switch that triggers dormant tumor cells to proliferate and initiate the brain metastatic cascade. ${ }^{26}$ Our demonstration of uPAR expression in the tumor, stromal, and endothelial cellular fractions of multiple tumor types also suggests that UPAR may play a fundamental role in canine brain tumorigenesis.

The malignant potential of human meningiomas and gliomas has been correlated with uPAR expression, which parallels our finding of significantly increased uPAR expression in canine high-grade gliomas compared with other tumor types and grades. ${ }^{1,8,13}$

Previous studies have shown that robust uPAR expression occurs at the invasive edges of malignant gliomas. ${ }^{25}$ In a limited number of cases, we observed intense UPAR immunoreactivity at the interface of GBM where it effaced the neuropil. The role of uPAR-mediated proteolytic cleavage in canine glioma invasion requires further investigation.

Investigations in human brain tumors have demonstrated that UPAR is a pharmacologically tractable molecular target for diagnostic and therapeutic purposes. . $^{12,27}$

The results of this study also indicate that uPAR is an attractive target for exploitation in dogs with spontaneous brain tumors, which are a faithful animal model of human disease. ${ }^{9,10}$ The results reported here served as the justification for our development of a recombinant uPAR-targeted oncolytic Newcastle disease virus (NDV) for use in canine brain tumors. By genetically modifying a native LaSota NDV strain as previously described, such that the LaSota fusion (F) protein cleavage site is replaced with a uPA cleavage site, ${ }^{28,29}$ we have generated the LaSota-uPA recombinant NDV strain the F protein of which is cleavable exclusively by uPAR. A Phase I clinical trial investigating LaSota-uPA in canine meningiomas has been initiated. ${ }^{30}$

\section{Conclusion}

We have shown that UPAR is overexpressed in multiple microenvironmental compartments in canine meningiomas, gliomas, and choroid plexus tumors. This study provides a rationale to develop and assess uPAR-targeted therapeutic strategies in naturally occurring canine brain tumors.

\section{Acknowledgments}

Portions of this study were funded by the Wallace H. Coulter Foundation Career Grant, the Virginia Biosciences Health Research Corporation, the Virginia Veterinary Memorial Fund, R01 CA139099/CA/NCI NIH HHS, and R21 AI070528/AI/NIAID NIH HHS. We acknowledge and appreciate the contributions of our friend and colleague, 
Dr Subbiah Elankumaran, who passed away on September 2, 2015 , prior to completion of the article.

\section{Disclosure}

The authors report no conflicts of interest in this work.

\section{References}

1. Liotta LA, Stetler-Stevenson WG. Tumor invasion and metastasis: an imbalance of positive and negative regulation. Cancer Res. 1991; 51(Suppl 18):5054-5059.

2. Noh H, Hong S, Huang S. Role of urokinase receptor in tumor progression and development. Theranostics. 2013;3(7):487-495.

3. Dano K, Andreasen PA, Grondahl-Hansen K, Kristensen P, Nielsen LS, Skriver L. Plasminogen activators, tissue degradation and cancer. $A d v$ Cancer Res. 1985;44:139-266.

4. Leonardi L, Quattrini I, Roperto F, Benassi MS. Protease expression in giant cell tumour of bone: a comparative study on feline and human samples. Res Vet Sci. 2013;95(2):310-315.

5. Santos A, Lopes C, Marques RM, et al. Immunohistochemical analysis of urokinase plasminogen activator and its prognosis in canine mammary tumors. Vet J. 2011;189(1):43-48.

6. Kwaaan HC, Mazar AP, McMahon BJ. The apparent uPA/PAI-1 paradox in cancer: more than meets the eye. Semin Thromb Hemost. 2013;39(4): 382-391.

7. Collen D, Lijnen HR. Basic and clinical aspects of fibrinolysis and thrombolysis. Blood. 1991;78(12):3114-3124.

8. Gilder AS, Jones JA, Hu J, et al. Soluble urokinase receptor is released selectively by glioblastoma cells that express epidermal growth factor receptor variant III and promotes tumor cell migration and invasion. J Biol Chem. 2015;290(24):14798-14809.

9. Debinski W, Dickinson PJ, Rossmeisl JH, Robertson J, Gibo D. New agents for targeting of IL-13RA2 expressed in primary human and canine brain tumors. PLoS One. 2013;8(10):e77719.

10. LeBlanc AK, Mazcko C, Brown DE, et al. Creation of an NCI comparative brain tumor consortium: informing the translation of new knowledge from canine to human brain tumor patients. Neuro Oncol. 2016; 18(9):1209-1218.

11. Mohan PM, Chintala SK, Mohanam S, et al. Adenovirus-mediated delivery of antisense gene to urokinase-type plasminogen activator suppresses glioma invasion and tumor growth. Cancer Res. 1999;59(14): 3369-3373.

12. Vallera DA, Li C, Jin N, Panoskaltsis-Mortari A, Hall WA. Targeting urokinase-type plasminogen activator receptor on human glioblastoma tumors with diphtheria toxin fusion protein DTAT. J Natl Cancer Inst. 2002;94(8):597-606

13. Kargiotis O, Chetty C, Gogineni V, et al. uPA/uPAR downregulation inhibits radiation-induced migration, invasion, and angiogenesis in IOMM-Lee meningioma cells and decreased tumor growth in vivo. Int J Oncol. 2008;33(5):937-947.

14. Rossmeisl JH, Robertson JL, Zimmerman KL, Higgins MA, Geiger DA. Cyclooxygenase-2 (COX-2) expression in canine intracranial meningiomas. Vet Comp Oncol. 2009;7(3):173-180.
15. Rossmeisl JH, Jones JC, Zimmerman KL, Robertson JL. Survival time following hospital discharge in dogs with palliatively treated primary brain tumors. J Am Vet Med Assoc. 2013;242(2):193-198.

16. Faleiro MR, Toledo DC, Rodrigues MMP, et al. uPAR expression in canine normal prostate and with proliferative disorders. Ci Anim Bras Goiania. 2013;14(2):237-244.

17. Untergasser A, Nijveen H, Rao X, Bisseling T, Geurts R, Leunissen JAM. Primer3Plus, an enhanced web interface to Primer3. Nucleic Acids Res. 2007;35:W71-W74.

18. Altschul SF, Madden TL, Schaffer AA, et al. Gapped BLAST and PSI-BLAST: a new generation of protein database search programs. Nucleic Acids Res. 1997;25(17):3389-3402.

19. Kim TD, Song KS, Li G, et al. Activity and expression of urokinasetype plasminogen activator and matrix metalloproteinases in human colorectal cancer. BMC Cancer. 2006;6:211.

20. Jankun J, Skrzypczak-Jankun E. Molecular basis of specific inhibition of urokinase plasminogen activator by amiloride. Cancer Biochem Biophys. 1999;17(1-2):109-123.

21. Dickinson PJ. Advances in diagnostic and treatment modalities for intracranial tumors. J Vet Int Med. 2014;28(4):1165-1185.

22. Song RB, Vite CH, Bradley CW, Cross JR. Postmortem evaluation of 435 cases of intracranial neoplasia in dogs and relationship of neoplasm with breed, age, and body weight. J Vet Intern Med. 2013;27(5): $1143-1152$.

23. Snyder JM, Shofer FS, Van Winkle TJ, Massicotte C. Canine intracranial primary neoplasia: 173 cases (1986-2003). J Vet Intern Med. 2006; 20(3):669-675.

24. Archinti M, Britto M, Eden G, Furlan F, Murphy R, Degryse B. The urokinase receptor in the central nervous system. CNS Neurol Disord Drug Targets. 2011;10(2):271-294.

25. Mohanam S, Sawaya RE, Yamamoto M, Bruner JM, Nicholson GL, Rao JS. Proteolysis and invasiveness of brain tumors: role of urokinase-type plasminogen activator receptor. $J$ Neurooncol. 1994;22(2): $153-160$.

26. Vishnoi M, Peddibhotla S, Yin W, et al. The isolation and characterization of CTC subsets related to breast cancer dormancy. Sci Rep. 2015; 5:17533.

27. Hirata K, Tamaki N. uPAR as a glioma imaging target. $J$ Nuc Med. 2016;57(2):169-170.

28. Kim SH, Wanasen N, Paldurai A, Xiao S, Collins PL, Samal SK. Newcastle disease virus fusion protein is the major contributor to protective immunity of genotype-matched vaccine. PLoS One. 2013; 8(8):e74022

29. Shobana R, Samal SK, Elankumaran S. Prostate-specific antigenretargeted recombinant Newcastle disease virus for prostate cancer virotherapy. J Virol. 2013;87(7):3792-3800.

30. Phase I Clinical Trial of Recombinant Newcastle Disease Virus for Canine Intracranial Meningiomas. Virginia: College of VirginiaMaryland Veterinary Medicine. Available from: http://www.vetmed. vt.edu/clinical-trials/current-studies/meningioma.asp. Accessed March $13,2016$.

\section{Dovepress}

\section{Publish your work in this journal}

OncoTargets and Therapy is an international, peer-reviewed, open access journal focusing on the pathological basis of all cancers, potential targets for therapy and treatment protocols employed to improve the management of cancer patients. The journal also focuses on the impact of management programs and new therapeutic agents and protocols on

Submit your manuscript here: http://www.dovepress.com/oncotargets-and-therapy-journal patient perspectives such as quality of life, adherence and satisfaction. The manuscript management system is completely online and includes a very quick and fair peer-review system, which is all easy to use. Visit http://www.dovepress.com/testimonials.php to read real quotes from published authors. 